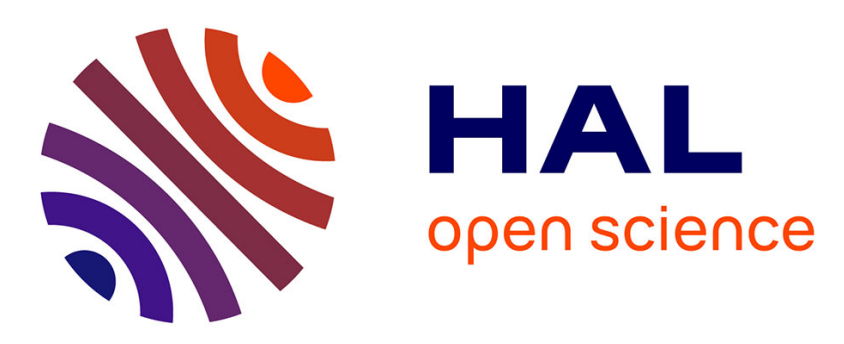

\title{
On the Rate and Energy Efficiency Comparison of Reconfigurable Intelligent Surfaces with Relays
}

Konstantinos Ntontin, Marco Di Renzo, Fotis Lazarakis

\section{To cite this version:}

Konstantinos Ntontin, Marco Di Renzo, Fotis Lazarakis. On the Rate and Energy Efficiency Comparison of Reconfigurable Intelligent Surfaces with Relays. IEEE International Workshop on Signal Processing Advances in Wireless Communications (SPAWC), May 2020, Atlanta (virtual ), United States. 10.1109/SPAWC48557.2020.9154308 . hal-03022567

\section{HAL Id: hal-03022567 https://hal.science/hal-03022567}

Submitted on 24 Nov 2020

HAL is a multi-disciplinary open access archive for the deposit and dissemination of scientific research documents, whether they are published or not. The documents may come from teaching and research institutions in France or abroad, or from public or private research centers.
L'archive ouverte pluridisciplinaire HAL, est destinée au dépôt et à la diffusion de documents scientifiques de niveau recherche, publiés ou non, émanant des établissements d'enseignement et de recherche français ou étrangers, des laboratoires publics ou privés. 


\title{
On the Rate and Energy Efficiency Comparison of Reconfigurable Intelligent Surfaces with Relays
}

\author{
Konstantinos Ntontin*, Marco Di Renzo ${ }^{\dagger}$, and Fotis Lazarakis* \\ *Institute of Informatics and Telecommunications, National Centre for Scientific Research "Demokritos", \\ 15310, Agia Paraskevi, Greece, E-mail:\{konstantinos.ntontin, flaz\}@iit.demokritos.gr \\ †Université Paris-Saclay, CNRS, CentraleSupélec, Laboratoire des Signaux et Systèmes, Gif-sur-Yvette, France, \\ E-mail: marco.direnzo@centralesupelec.fr
}

\begin{abstract}
In this paper, we perform a performance comparison, in terms of achievable rate and energy efficiency, of a reconfigurable intelligent surface (RIS)-aided nextgeneration fronthaul network operating in the $D$ band with its relay-aided counterpart. For their energy efficiency comparison, the insertion losses and power consumption of the electronic components related with the deployed nodes is taken into account. Numerical results show that the RIS-aided network outperforms the relay-aided one, both in terms of rate and energy efficiency, only for adequately large RISs.
\end{abstract}

Index Terms-D band, reconfigurable intelligent surfaces, relays, rate, energy efficiency.

\section{INTRODUCTION}

Data-rate demands have been exponentially increasing over the last years, which have made current mobile-access networks relying on sub-6 GHz bands, such as the Long Term Evolution (LTE) standard, reach their limits in terms of available bandwidth. Due to such a bottleneck, the migration to above- $6 \mathrm{GHz}$ bands that allow the use of much larger bandwidths is envisioned for $5 \mathrm{G}$ and beyond networks. Regarding the potential of the above- $6 \mathrm{GHz}$ bands for wireless communication, bands in the lower-end millimeter-wave (mmWave) spectrum are currently used for point-to-point and point-tomultipoint line-of-sight (LOS) wireless backhaul/fronthaul and fixed-wireless access networks [1]. Such deployments mainly span the $30-100 \mathrm{GHz}$ range.

Furthermore, the expected migration of future mobile-access networks to the $30-100 \mathrm{GHz}$ range is inevitably going to move the corresponding wireless backhaul/fronthaul links towards the beyond-100 $\mathrm{GHz}$ bands in order to accommodate the increased user data rates. More specifically, several vendors have performed LOS trials in the D band that corresponds to the 130-174.8 GHz spectrum, which showcase the feasibility and potential of using it for wireless communication in such networks [2]. However, although NLOS communication, as aforementioned, is feasible in the $30-100 \mathrm{GHz}$ range, the higher propagation loss of beyond-100 GHz bands is likely to render such communication infeasible.

With respect to the expected NLOS bottleneck of future beyond-100 GHz wireless backhaul/fronthaul networks, the conventional way of circumventing a NLOS link is by providing alternative LOS routes through relay nodes. Although such an approach is a well established method to increase the coverage in poor quality direct connections, it is argued that it is unlikely to constitute a viable approach for very high-frequency networks. This is due to the increased power consumption of the active RF components in such frequencies that would be involved in a massive network deployment [3]. Recently, an alternative to relaying approach, which is based on the use of reconfigurable intelligent surfaces (RISs), was introduced for communication purposes [4]. RISs consist of metasurfaces, which are artificial surfaces comprising a number of sub-wavelength radiating elements, named unit cells, embedded on a dielectric substrate with thickness much smaller than the wavelength. According to the arrangement, structure, and size of the unit cells, metasurfaces can perform a variety of functions on the impinging waves, such as reflection and refraction in directions that do not obey to the classical Snell's law, absorption, and polarization change. Contrary to static metasurface designs, functionality of RISs can dynamically change based on external stimuli, according to which parameters such as the phase response and the size of the unit cells are altered. This is enabled by switching elements, such as PIN diodes, radio frequency (RF)-microelectromechanical systems, and varactor diodes, that are introduced between adjacent unit cells [4].

As far as the performance comparison of RISs with relays is concerned, in [5] the authors show that an RIS acting as reflector can be up to $300 \%$ more energy efficient than an amplify-and-forward relay. However, the RF electronics model that the authors use neither takes into account the insertion loss of the switching elements that are used for phase shifting, which can reach several $\mathrm{dBs}$ according to the literature [6], nor the insertion loss corresponding to common RF electronic components needed, such as power combiners and dividers. Contrary to [5], in [7] the authors show that an RIS needs hundred of radiating elements to outperform, in terms of rate, a single-antenna half-duplex (HD) and decodeand-forward relay. In addition, they show that an RIS can be more energy efficient than the relay only for very high data rates. Furthermore, the RF electronics model used is the same as in [5]. Hence, the insertion loss of common RF circuitry 


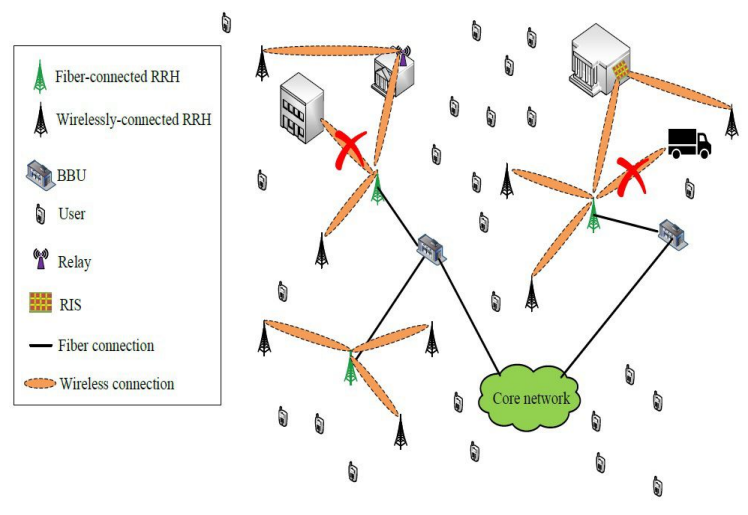

Fig. 1: Network layout.

needed, such as phase shifters and power combiners and dividers, is not included. Finally, in [8] the authors consider only the rate comparison of an RIS with both a HD and a full-duplex relay and show that a sufficiently large RIS offers the same rate performance with a single-antenna relay when the former is used for anomalous reflection instead of beamforming/focusing.

Contribution: Motivated by the drawbacks of previous works regarding the performance comparison of RISs with relays, such as their conflicting outcomes, the considered simplistic RF electronics model that does not take into account the insertion losses of the needed RF components, and the lack of such a comparison in a network setup, in this work we consider a next-generation fronthaul network operating in the D band, which consists of point-to-point fiber and wireless links that can be either LOS or NLOS. In the latter case, the communication is assisted by either multi-element RISs acting as reflectors or multiple-antenna HD and amplify-and-forward (AF) relays. Furthermore, By considering a realistic power consumption model for the deployed nodes, we formulate the network achievable rate and energy efficiency expressions when the network is either RIS or relay assisted.

The rest of this work is structured as follows: In Section II, the system model is presented, whereas in Section III the power consumption model of the involved nodes and the formulation of the network achievable rate and energy efficiency are introduced. Numerical results are provided in Section IV and, finally, Section V concludes this work.

\section{SYSTEM AND POWER CONSUMPTION MODELS}

\section{A. System model}

The system model assumptions are the following:

1) Network layout: The network layout is depicted in Fig. 1. We assume a Cloud-RAN downlink fronthaul architecture in which the deployed remote radio heads (RRHs), which contain only the RF circuitry and antennas, are associated with baseband processing units (BBUs) in which the baseband processing is performed. As far as the RRHs are concerned, we assume that their position and their number is described by a uniform Poisson point process on $\mathbb{R}^{2}$, denoted by $\Phi_{R R H}$, with intensity $\lambda_{R R H}=1 /\left(\pi r_{R R H}^{2}\right)$, where $r_{R R H}$ is the average inter-RRH distance. In addition, due to the high cost of fiberconnecting every RRH with its associated BBU, we assume that a portion of the RRHs are connected to their respective BBUs by wirelessly connecting to RRHs, either directly or through an RIS or relay, that are fiber-connected to the same BBUs. Hence, we consider that the fiber-connected RRHs are described by the PPP process $\Phi_{R R H}^{(C)}$ with intensity $\lambda_{R R H}^{(C)}$ and the wirelessly-connected ones by the PPP process $\Phi_{R R H}^{(W)}$ with intensity $\lambda_{R R H}^{(W)}$. Consequently, it holds that $\Phi_{R R H}=$ $\Phi_{R R H}^{(C)} \cup \Phi_{R R H}^{(W)}$ and $\lambda_{R R H}=\lambda_{R R H}^{(C)}+\lambda_{R R H}^{(W)}$.

2) Association rule: We assume that the wirelesslyconnected RRHs are associated with their closest fiberconnected RRH.

3) Propagation conditions: We consider that a portion of the wireless RRH connections, which we denote by $p_{L O S}$, is subject to LOS conditions, whereas the remaining RRHs, with portion $p_{N L O S}=1-p_{L O S}$, are subject to NLOS conditions due to large objects, such as buildings and vehicles. For the wireless-RRH links that are subject to NLOS conditions, the communication is realized through 2-hop LOS links originating from RISs acting as reflectors or relays that are mounted on neighbouring tall structures, such as buildings.

4) RIS/relay placement: Let us denote the distance between a transmit and a receive RRH by $d_{T x-R x}$. We assume that the RIS or relay that assists the communication can be uniformly located inside a circle with radius $\kappa_{R} d_{T x-R x}$, where $\kappa_{R} \in \mathbb{R}$ and center the middle of $d_{T x-R x}$.

5) Relay communication principle: The communication is realized in 2 time slots. In the first slot, only the communication between the transmit RRH and the relay is realized, whereas the receive RRH remains silent. In the second time slot, the relay-receive RRH communication is realized and the transmit RRH remains silent. In addition, we assume that the relays use the same set of antennas for transmitting and receiving.

6) Type and gain of the RRH and relay antennas: We assume that for transmission and reception the RRHs and relays are equipped with half-wavelength dipole antennas that are planarly arranged. By assuming that the efficiency of the antenna arrays is equal to 1 and the number of antennas in the $\mathrm{x}$ - and $\mathrm{y}$-axis of either the RRHs or relays is equal to $M_{x}$ and $M_{y}$, respectively, their gain $G_{A}$ is given by [9]

$$
G_{A}=\frac{4 \pi}{\int_{0}^{2 \pi} \int_{0}^{\pi} G_{d i p}^{2}(\theta, \varphi) A_{M_{x}}^{2}(\theta, \varphi) A_{M_{y}}^{2}(\theta, \varphi) \sin (\theta) d \theta d \varphi},
$$

where $G_{d i p}(\theta, \varphi)=\frac{\cos \left(\frac{\pi}{2} \sin (\theta) \cos (\varphi)\right)}{\sqrt{1-\sin ^{2}(\theta) \cos ^{2}(\varphi)}}$ is the element pattern of a half-wavelength dipole antenna and [9]

$$
\begin{aligned}
& A_{M_{x}}(\theta, \varphi)=\frac{\sin \left(M_{x} \frac{\pi}{2} \sin (\theta) \cos (\varphi)\right)}{M_{x} \sin \left(\frac{\pi}{2} \sin (\theta) \cos (\varphi)\right)} \\
& A_{M_{y}}(\theta, \varphi)=\frac{\sin \left(M_{y} \frac{\pi}{2} \sin (\theta) \sin (\varphi)\right)}{M_{y} \sin \left(\frac{\pi}{2} \sin (\theta) \sin (\varphi)\right)} .
\end{aligned}
$$


7) Size and structure of the RISs: We consider that the RISs consist of $N_{R I S}$ radiating elements planarly arranged. As far as their radiation pattern is concerned, which we denote by $G_{R I S}(\omega)$, where $\omega$ is the angle measured from the RIS broadside, due to the fact that the RIS radiating elements are envisaged to be electrically small and of low gain, we use the following model [10]:

$$
G_{R I S}(\omega)=\left\{\begin{array}{lc}
\gamma \cos ^{2 q}(\omega), & 0 \leq \omega<\pi / 2 \\
0, & p i / 2 \leq \omega<\pi
\end{array},\right.
$$

where $\gamma$ is a coefficient so that the power conservation is satisfied and $q$ is the gain of each element. If the physical area of the RIS is required to be equal to the sum of the effective aperture of the radiating elements as a design requirement, then for a distance between adjacent elements equal to half wavelength it is proved in [10] that $\gamma=\pi$ and $q=q_{0}=0.285$, which are the considered values in this work, without loss of generality.

As far as the size of the RISs is concerned, by considering far-field operation we assume that it is equal to $\alpha_{R} S_{R I S}$, where $\alpha_{R} \in(0,1]$, and $S_{R I S}$ is the size of the RIS such that both the transmit and receive RRHs of the respective RISassisted links are in the border of the near/far field region of the RIS. By denoting the transmit RRH-RIS and the RIS-receive RRH distances by $d_{T x-R I S}$ and $d_{R I S-R x}$, respectively, from theory it holds that [9]:

$$
S_{R I S}=v \min \left(d_{T x-R I S}, d_{R I S-R x}\right) / 2,
$$

where $v=c_{0} / f_{c}$ is the wavelength, $c_{0}$ is the speed of light, and $f_{c}$ is the carrier frequency. Considering a half-wavelength distance between adjacent unit cells, it holds that $N_{R I S}=$ $S_{R I S} /(v / 2)^{2}$.

8) Access protocol: A time-division multiple access protocol is considered for the communication of a fiber-connected RRH with its associated wirelessly connected RRHs.

9) Intersite interference: Due to the high directionality needed in beyond-100 GHz networks to overcome the large free-space path loss, we consider the network being noiselimited.

10) Noise at the receivers: We assume that the received signals are subject to additive white Gaussian noise. Its spectral density in $\mathrm{dBm}$, denoted by $N_{0}$, is equal to $-174+$ $10 \log _{10}(B W)+\mathcal{F}_{\mathrm{dB}}$, where $\mathcal{F}_{\mathrm{dB}}$ is the noise figure in $\mathrm{dB}$ and $B W$ is the transmission bandwidth.

11) Shadowing and fast fading: We consider that the network is not subject to shadowing and fast-fading conditions due to the fixed position of the nodes.

12) Height RIS/relay nodes: We assume that the RIS/Relay nodes are placed $h_{R}$ higher than the RRH nodes.

13) Power budget at the transmitting RRHs and relays: We assume that the the amplification units of the transmitting RRHs and the relays are adjusted so that both units produce an output power that is equal to $P_{t x}$.
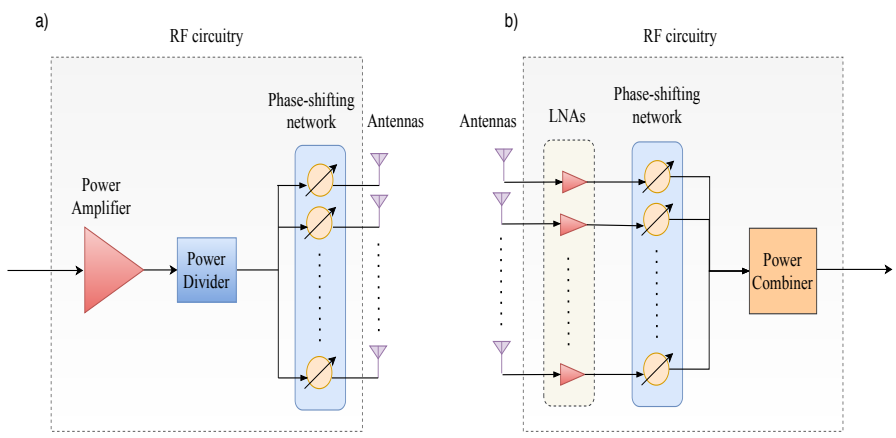

Fig. 2: Main RF circuitry used for a) transmission and b) reception in RRHs and relays.

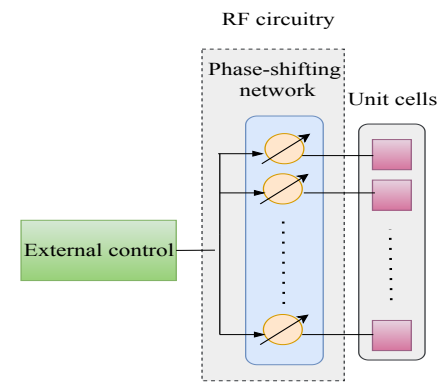

Fig. 3: Main RF circuitry of RISs.

14) Beamforming type: We consider that a purely analog transmit and receive beamforming is performed at both the RRHs and relays.

\section{Power consumption model, achievable Rate, AND ENERGY EFFICIENCY}

In this section, we first present the power consumption model related to the RRHs, relays, and RISs. Subsequently, we provide the mathematical formulation of the achievable rate and energy efficiency of the wireless fronthaul network in the RIS- and relay-assisted cases.

\section{A. Power consumption model}

Regarding the power consumption model, let us first present the RF electronics that equip the RRHs, relays, and RISs and consume the largest amount of power. As far as the RRHs and relays are concerned, the RF circuitry used for transmission and reception is depicted in Fig. 2a) and Fig. 2b), respectively. The power combiner and divider are passive devices that introduce insertion losses, denoted by $L_{C}$ and $L_{D}$, respectively. By assuming that 2-way components are used for power combining and dividing, where each component introduces a loss of $L_{C}^{(2-w a y)}$ and $L_{D}^{(2-w a y)}$, respectively, and that the total number of antennas of either the RRHs or the relays is equal to $M_{x} M_{y}$, it holds that $L_{C}=\left\lceil\log _{2}\left(M_{x} M_{y}\right)\right\rceil L_{C}^{(2-w a y)}$ and $L_{D}=\left\lceil\log _{2}\left(M_{x} M_{y}\right)\right\rceil L_{D}^{(2-w a y)}$, where \lceil\rceil denotes the next larger integer that is greater than $\mathrm{x}$. In addition, we assume that the phase shifters are active devices that introduce power consumption and insertion losses denoted by $P_{P S}$ and $L_{P S}$, respectively. As far as the RISs are concerned, the only 
circuitry required for their operation are the RF phase-shifters used for modifying the phase response of each unit cell. Such a model is depicted in Fig. 3. Without loss of generality, we assume that the phase shifters used in RRHs, relays, and RISs are the same. Hence, they exhibit the same power consumption and insertion losses. Regarding the total power consumption, we distinguish the following 3 cases:

1) Direct-link LOS communication: In the case that the communication between the 2 RRHs is realized through a direct LOS link, the total power consumption, which we denote by $P_{t o t}^{(L O S)}$, is given by

$$
P_{t o t}^{(L O S)}=\frac{P_{t x}}{\eta_{A}}+M_{x}^{(R R H)} M_{y}^{(R R H)}\left(2 P_{P S}+P_{L N A}\right),
$$

where $P_{L N A}$ is the power consumption of one low-noise amplifier (LNA) unit, $\eta_{A}$ is the efficiency of the power amplifier used at the RRHs, and $M_{x}^{(R R H)}$ and $M_{y}^{(R R H)}$ are the number of antennas in the x-axis and y-axis of the RRHs, respectively.

2) RIS-assisted communication: In the case that the communication between the RRHs is realized via an RIS, the total power consumption, which we denote by $P_{t o t}^{(R I S)}$, is given by

$P_{t o t}^{(R I S)}=\frac{P_{t x}}{\eta_{A}}+M_{x}^{(R R H)} M_{y}^{(R R H)}\left(2 P_{P S}+P_{L N A}\right)+N_{R I S} P_{P S}$.

3) Relay-assisted communication: In the case that the communication between the 2 RRHs is realized through a relay, the total power consumption in two time slots (since the communication is realized in two times slots due to the HD relay constraint), which we denote by $P_{\text {tot }}^{(\text {relay })}$, is given by

$$
\begin{aligned}
P_{\text {tot }}^{(\text {relay })} & =P_{t x}\left(\frac{1}{\eta_{A}}+\frac{1}{\eta_{R}}\right)+M_{x}^{(R R H)} M_{y}^{(R R H)}\left(2 P_{P S}+P_{L N A}\right) \\
& +M_{x}^{(\text {relay })} M_{y}^{(\text {relay })}\left(2 P_{P S}+P_{L N A}\right),
\end{aligned}
$$

where $\eta_{R}$ is the efficiency of the power amplifier used at the relays and $M_{x}^{(\text {relay })}$ and $M_{y}^{(\text {relay })}$ are their number of antennas in the $\mathrm{x}$-axis and $\mathrm{y}$-axis, respectively. Hence, for the average power consumption per time slot, which we denote by $P_{\text {tot }}^{(\text {relot } l o t}$, it holds that $P_{\text {tot }}^{(\text {relot }}=P_{\text {tot }}^{(\text {relay })} / 2$.

\section{B. Achievable rate}

Regarding the achievable rate, we distinguish the two cases of RIS-assisted and relay-assisted communication.

1) RIS-assisted communication: For the achievable rate of the RIS-assisted communication, denoted by $\bar{R}_{R I S}$, it holds

$$
\bar{R}_{R I S}=p_{L O S} \bar{R}_{L O S}+p_{N L O S} \bar{R}_{N L O S}^{(R I S)}
$$

where $\bar{R}_{L O S}=E\left\{\frac{B W}{N_{R R H}} \log _{2}\left(1+S N R_{L O S}\right)\right\}$ and $\bar{R}_{N L O S}^{(R I S)}=E\left\{\frac{B W}{N_{R R H}} \log _{2}\left(1+S N R_{N L O S}^{(R I S)}\right)\right\}$ are the av- erage rates of the LOS communication and communication through the RISs in the case of NLOS direct links, respectively. $N_{R R H}$ is the number of wireless RRHs associated with the particular fiber-connected RRH. In addition, it holds that

$$
S N R_{L O S}=\frac{P_{t x}\left(G_{A}^{(R R H)}\right)^{2} G_{L N A}}{\left(L_{P S}\right)^{2} L_{C} L_{D} k_{0} d_{T x-R x}^{2} N_{0}},
$$

where $k_{0}=(4 \pi / v)^{2}$ and $G_{A}^{(R R H)}$ and $G_{L N A}$ are the RRH antenna and LNA gains, respectively. Regarding $S N R_{N L O S}^{(R I S)}$, which is the received SNR, by considering that the phase response of the unit cells is adjusted so that the departing waves are coherently summed in the receiver (beamforming case), following the steps in [10] it holds that

$$
S N R_{N L O S}^{(R I S)}=\frac{P_{t x}\left(G_{A}^{(R R H)}\right)^{2} G_{L N A}\left(\alpha_{R} S_{R I S}\right)^{2} \alpha\left(\theta_{i}, \theta_{d}\right) A}{\left(L_{P S}\right)^{3} L_{C} L_{D} 16 \pi^{2} d_{T x-R I S}^{2} d_{R I S-R x}^{2} N_{0}},
$$

where $\alpha\left(\theta_{i}, \theta_{d}\right)=\left(\cos \left(\theta_{i}\right) \cos \left(\theta_{d}\right)\right)^{2 q_{0}} . \theta_{i}$ and $\theta_{d}$ are the incidence and departure angles, respectively, and $A$ is the power reflection coefficient for each unit cell.

2) Relay-assisted communication: For the achievable rate $\bar{R}_{\text {relay }}$ of the relay-assisted communication, it holds that

$$
\bar{R}_{\text {relay }}=p_{L O S} \bar{R}_{L O S}+p_{N L O S} \bar{R}_{N L O S}^{(\text {relay })},
$$

where $\bar{R}_{N L O S}^{(\text {relay })}=0.5 E\left\{\frac{B W}{N_{R R H}} \log _{2}\left(1+S N R_{N L O S}^{(\text {relay })}\right)\right\}$. It holds that

$$
S N R_{N L O S}^{(\text {relay })}=\frac{\frac{P_{t x}\left(G_{A}^{(R R H)}\right)^{2}\left(G_{A}^{(\text {relay })}\right)^{2}\left(G_{L N A}\right)^{2} G_{a m p}^{(\text {relay })}}{\left(L_{P S}\right)^{4}\left(L_{C}\right)^{2}\left(L_{D}\right)^{2} k_{0}^{2} d_{T x-\text { relay }}^{2} d_{\text {relay }-R x}^{2}}}{N_{0}\left(\frac{G_{A}^{(R R H)} G_{A}^{(r e l a y)} G_{L N A} G_{a m p}^{(r e l a y)}}{\left(L_{P S}\right)^{2} L_{C} L_{D} k_{0} d_{\text {relay }-R x}^{2}}+1\right)},
$$

where $G_{A}^{(r e l a y)}$ is the antenna gain of the relays and $G_{a m p}^{(r e l a y)}$ is the relay amplification gain, given by

$$
G_{a m p}^{(\text {relay })}=\frac{P_{t x}}{\frac{P_{t x} G_{A}^{(R R H)} G_{A}^{(\text {relay })} G_{L N A}}{\left(L_{P S}\right)^{2} L_{C} L_{D} k_{0} d_{T x-\text { relay }}^{2}}+N_{0}} .
$$

\section{Energy efficiency}

1) RIS-assisted communication: For the energy efficiency $\bar{E}_{R I S}$ of the RIS-assisted communication, it holds that

$$
\begin{aligned}
\bar{E}_{R I S} & =p_{L O S} E\left\{\frac{\frac{B W}{N_{R R H}} \log _{2}\left(1+S N R_{L O S}\right)}{P_{t o t}^{(L O S)}}\right\} \\
& +p_{N L O S} E\left\{\frac{\frac{B W}{N_{R R H}} l_{2}\left(1+S N R_{N L O S}^{(R I S)}\right)}{P_{t o t}^{(R I S)}}\right\} .
\end{aligned}
$$

2) Relay-assisted communication: For the energy efficiency of the relay-assisted communication, which we denote by $\bar{E}_{\text {relay }}$, it holds that 


$$
\begin{aligned}
\bar{E}_{\text {relay }} & =p_{L O S} E\left\{\frac{\frac{B W}{N_{R R H}} \log _{2}\left(1+S N R_{L O S}\right)}{P_{\text {tot }}^{(L O S)}}\right\} \\
& +p_{N L O S} 0.5 E\left\{\frac{\frac{B W}{N_{R R H}} \log _{2}\left(1+S N R_{N L O S}^{(\text {relay })}\right)}{P_{\text {tot }}^{(\text {relay })}}\right\} .
\end{aligned}
$$

\section{NUMERICAL RESUlts}

For the parameter values of Table I and a high and a low value of $L_{P S}$, Fig. 4 illustrates the performance comparison of the RIS- and relay-assisted networks versus the average dimension in wavelengths of each of the two sides of the RISs and relays. The considered range corresponds to $\alpha_{R}$ in the range $[0.02,1]$. For a fair comparison of the two networks, we assume that the RISs and relays have the same number of radiating elements with inter-element distance equal to $v / 2$.

TABLE I: Parameter values used in the simulations.

\begin{tabular}{|c|c|}
\hline$r_{R R H}, \lambda_{R R H}^{(C)}$ & $150 \mathrm{~m}, \lambda_{R R H} / 4$ \\
\hline$P_{t x}, f_{c}$ & $1 \mathrm{~W}, 140 \mathrm{GHz}$ \\
\hline$B W, p_{N L O S}$ & $4 \mathrm{GHz}, 0.4$ \\
\hline$\kappa_{R}, \mathcal{F}_{\mathrm{dB}}$ & $1,10 \mathrm{~dB}$ \\
\hline$\theta_{i}, \theta_{d}$ & uniform in $(0, \pi / 3)$ \\
\hline$h_{R}, A$ & $8 \mathrm{~m}, 0.9$ \\
\hline$M_{x}^{(R R H)}, M_{y}^{(R R H)}$ & 128 \\
\hline$\eta_{A}, \eta_{R}$ & 0.1 \\
\hline$L_{C}^{(2-w a y)}, L_{D}^{(2-\text { way })}$ & $0.5 \mathrm{~dB}$ \\
\hline$P_{P S}, P_{L N A}, G_{L N A}$ & $10 \mathrm{~mW}, 40 \mathrm{~mW}, 20 \mathrm{~dB}$ \\
\hline
\end{tabular}

As we observe from Fig. 4, there is a threshold value regarding the dimensions of RIS and relays above which the RISassisted network outperforms its relay-assisted counterpart in terms of both achievable rate and energy efficiency. The higher $L_{P S}$ is, the higher that threshold is. The threshold occurs due to the fact that the SNR of the RIS-assisted links scales with the squared power of the number of radiating elements, according to (11), whereas it can be proved that the SNR of the relay-assisted links, according to (13), scales linearly with the number of elements.

\section{CONCLUSIONS}

We have conducted this work to give an answer on whether RISs should be prefered over relays in a realistic network setup. Towards this, we have considered a next-generation fronthaul network operating in the D band and included in the modeling the insertion losses and power consumption of the electronic components of the involved nodes.

The numerical results have revealed that for sufficiently large RISs the RIS-assisted network outperforms its relayassisted counterpart in terms of both achievable rate and energy efficiency, even for a high value of the insertion loss of the phase-shifting components.

\section{ACKNOWLEDGEMENTS}

This work has received funding from the H2020 Artificial Intelligence Aided D-Band Network for 5G Long Term Evolution (ARIADNE) project under Grant Agreement 871464.

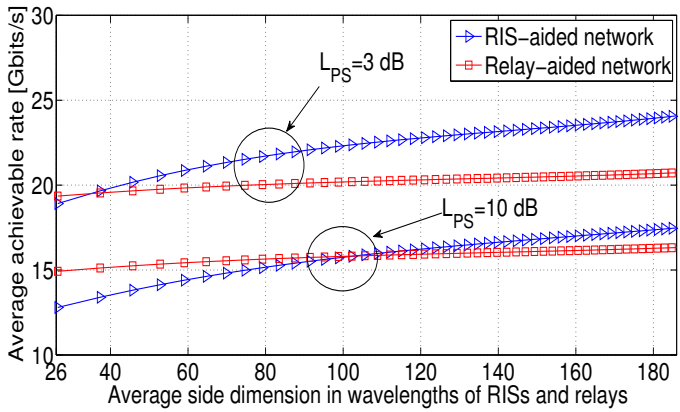

(a) Average achievable rate.

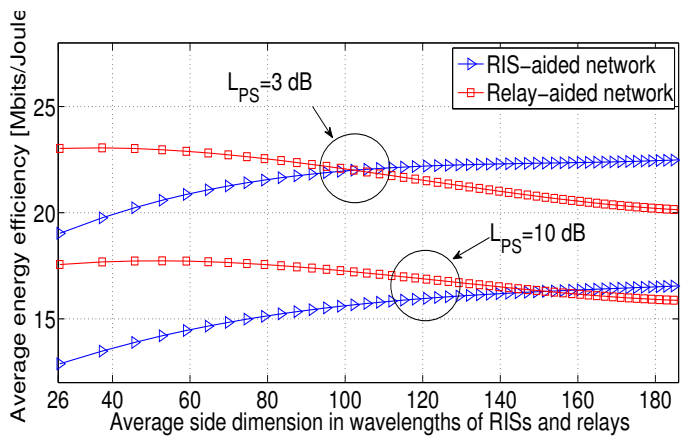

(b) Average energy efficiency.

Fig. 4: Performance comparison of the RIS-aided and relay-aided networks.

The authors would also like to cordially thank the anonymous reviewers for their suggestions for improving this work.

\section{REFERENCES}

[1] "Mobile backhaul options: Spectrum analysis and recommendations," ABI Research, Tech. Rep., Sep. 2018.

[2] "Millimetre wave transmission (mwt); analysis of spectrum, license schemes and network scenarios in the d-band," ETSI, Tech. Rep., Aug. 2018.

[3] W. Khawaja, O. Ozdemir, Y. Yapici, F. Erden, M. Ezuma, and I. Guvenc, "Coverage Enhancement for NLOS mmwave Links Using Passive Reflectors," arXiv:1905.04794.

[4] E. Basar, M. D. Renzo, J. de Rosny, M.-S. Alouini, and R. Zhang, "Wireless Communications Through Reconfigurable Intelligent Surfaces," IEEE Access, vol. 7, pp. 116753-116773, 2019.

[5] C. Huang, A. Zappone, G. C. Alexandropoulos, M. Debbah, and C. Yuen, "Wireless Communications Through Reconfigurable Intelligent Surfaces," IEEE Trans. Wirel. Commun., vol. 18, no. 8, pp. 4157-4170, Aug. 2019.

[6] B. Biglarbegian, M. R. Nezhad-Ahmadi, M. Fakharzadeh, and S. SafaviNaeini, "Millimeter-Wave Reflective-Type Phase Shifter in Cmos Technology," IEEE Microwave and Wireless Components Letters, vol. 19, no. 9, pp. 560-562, Sep. 2009.

[7] E. Bjornson, O. Ozdogan, and E. G. Larsson, "Intelligent Reflecting Surface vs. Decode-and-Forward: How Large Surfaces Are Needed to Beat Relaying?" IEEE Wirel. Commun. Lett., to appear.

[8] M. D. Renzo, K. Ntontin, J. Song, F. H. Danufane, X. Qian, F. Lazarakis, J. de Rosny, D.-T. Phan-Huy, O. Simeone, R. Zhang, M. Debbah, G. Lerosey, M. Fink, S. Tretyakov, and S. Shamai (Shitz), "Reconfigurable Intelligent Surfaces vs. Relaying: Differences, Similarities, and Performance Comparison," arXiv:1908.08747.

[9] C. A. Balanis, Antenna Theory: Analysis and Design, 3rd ed. John Wiley, 2007.

[10] S. W. Ellingson, "Path Loss in Reconfigurable Intelligent SurfaceEnabled Channels," arXiv:1912.06759. 